\title{
Optimization of the in Vitro and in Vivo
}

\author{
Properties of a Novel Series of 2,4,5-
}

\section{Trisubstituted Imidazoles as Potent $\mathrm{CCK}_{2}$}

\section{Antagonists}

Ildiko M. Buck*, James W. Black, Tracey Cooke, David J. Dunstone, John D. Gaffen, Eric P. Griffin, Elaine A. Harper, Robert A. D. Hull, S. Barret Kalindjian, Elliot J. Lilley, Ian D. Linney, Caroline M. R. Low, Iain M. McDonald, Michael J. Pether, Sonia P. Roberts, Nigel P. Shankley, Mark E. Shaxted, Katherine I. M. Steel, David A. Sykes, Matthew J. Tozer, Gillian F. Watt, Martin K. Walker, Laurence Wright, Paul T. Wright.

\section{Appendix}

$\underline{\text { Analytical data of compounds used in biological tests }}$

\begin{tabular}{|l|l|l|l|}
\hline Compound & Found & Formula & Required \\
\hline $\mathbf{1 3}$ & $\mathrm{C} 63.88 ;$ & $\mathrm{C}_{30} \mathrm{H}_{32} \mathrm{~N}_{2} \mathrm{O}_{3} \bullet \mathrm{C}_{7} \mathrm{H}_{17} \mathrm{NO}_{5} \bullet 1.6 \mathrm{H}_{2} \mathrm{O}$ & $\mathrm{C} 64.15 ;$ \\
& $\mathrm{H} \mathrm{7.32;}$ & & $\mathrm{H} 7.60 ;$ \\
& $\mathrm{N} 5.82 \%$ & & $\mathrm{~N} 6.06 \%$. \\
\hline $\mathbf{1 4}$ & $\mathrm{C} 64.72 ;$ & $\mathrm{C}_{31} \mathrm{H}_{34} \mathrm{~N}_{2} \mathrm{O}_{3} \bullet \mathrm{C}_{7} \mathrm{H}_{17} \mathrm{NO}_{5} \bullet 1.5 \mathrm{H}_{2} \mathrm{O}$ & $\mathrm{C} 64.72 ;$ \\
& $\mathrm{H} \mathrm{7.77;}$ & & $\mathrm{H} 7.72 ;$ \\
& $\mathrm{N} 5.87 \%$ & & N 5.96\% \\
\hline
\end{tabular}




\begin{tabular}{|c|c|c|c|}
\hline 21 & $\begin{array}{l}\text { C 60.48; } \\
\text { H 7.84; } \\
\text { N 8.04\% }\end{array}$ & $\mathrm{C}_{29} \mathrm{H}_{31} \mathrm{~N}_{3} \mathrm{O}_{3} \bullet \mathrm{C}_{7} \mathrm{H}_{17} \mathrm{NO}_{5} \bullet 2.9 \mathrm{H}_{2} \mathrm{O}$ & $\begin{array}{l}\text { C 60.35; } \\
\text { H 7.56; } \\
\text { N 7.82\% }\end{array}$ \\
\hline 22 & $\begin{array}{l}\text { C 61.98; } \\
\text { H 7.63; } \\
\text { N 7.82\% }\end{array}$ & $\mathrm{C}_{30} \mathrm{H}_{33} \mathrm{~N}_{3} \mathrm{O}_{3} \bullet \mathrm{C}_{7} \mathrm{H}_{17} \mathrm{NO}_{5} \bullet 2.1 \mathrm{H}_{2} \mathrm{O}$ & $\begin{array}{l}\text { C 61.98; } \\
\text { H 7.63; } \\
\text { N } 7.81 \%\end{array}$ \\
\hline 23 & $\begin{array}{l}\text { C 57.68; } \\
\text { H 7.60; } \\
\text { N 9.16\% }\end{array}$ & $\mathrm{C}_{23} \mathrm{H}_{25} \mathrm{~N}_{3} \mathrm{O}_{3} \bullet \mathrm{C}_{7} \mathrm{H}_{17} \mathrm{NO}_{5} \bullet 2.0 \mathrm{H}_{2} \mathrm{O}$ & $\begin{array}{l}\text { C 57.85; } \\
\text { H 7.45; } \\
\text { N 8.99\%. }\end{array}$ \\
\hline 24 & $\begin{array}{l}\text { C 58.04; } \\
\text { H 7.66; } \\
\text { N } 8.51 \%\end{array}$ & $\mathrm{C}_{24} \mathrm{H}_{27} \mathrm{~N}_{3} \mathrm{O}_{3} \bullet \mathrm{C}_{7} \mathrm{H}_{17} \mathrm{NO}_{5} \bullet 2.3 \mathrm{H}_{2} \mathrm{O}$ & $\begin{array}{l}\text { C 57.87; } \\
\text { H 7.64; } \\
\text { N 8.71\% }\end{array}$ \\
\hline 25 & $\begin{array}{l}\text { C 60.17; } \\
\text { H 7.75; } \\
\text { N } 8.68 \%\end{array}$ & $\mathrm{C}_{26} \mathrm{H}_{29} \mathrm{~N}_{3} \mathrm{O}_{3} \bullet \mathrm{C}_{7} \mathrm{H}_{17} \mathrm{NO}_{5} \bullet 1.7 \mathrm{H}_{2} \mathrm{O}$ & $\begin{array}{l}\text { C } 60.34 ; \\
\text { H } 7.57 ; \\
\text { N } 8.53 \%\end{array}$ \\
\hline 26 & $\begin{array}{l}\text { С 61.26; } \\
\text { Н 6.86; } \\
\text { N } 8.47 \%\end{array}$ & $\mathrm{C}_{26} \mathrm{H}_{23} \mathrm{~N}_{3} \mathrm{O}_{3} \bullet \mathrm{C}_{7} \mathrm{H}_{17} \mathrm{NO}_{5} \bullet 1.5 \mathrm{H}_{2} \mathrm{O}$ & $\begin{array}{l}\text { C 61.11; } \\
\text { H 6.70; } \\
\text { N 8.54\% }\end{array}$ \\
\hline 27 & $\begin{array}{l}\text { C 61.88; } \\
\text { H 7.42; } \\
\text { N } 8.05 \%\end{array}$ & $\mathrm{C}_{29} \mathrm{H}_{31} \mathrm{~N}_{3} \mathrm{O}_{3} \bullet \mathrm{C}_{7} \mathrm{H}_{17} \mathrm{NO}_{5} \bullet 1.9 \mathrm{H}_{2} \mathrm{O}$ & $\begin{array}{l}\text { C } 61.92 ; \\
\text { H } 7.47 ; \\
\text { N } 8.02 \%\end{array}$ \\
\hline 28 & $\begin{array}{l}\text { C } 76.43 ; \\
\text { H } 8.14 ; \\
\text { N } 8.76 \%\end{array}$ & $\mathrm{C}_{30} \mathrm{H}_{35} \mathrm{~N}_{3} \mathrm{O} \bullet \mathrm{H}_{2} \mathrm{O}$ & $\begin{array}{l}\text { C } 76.40 ; \\
\text { H 7.91; } \\
\text { N } 8.91 \%\end{array}$ \\
\hline 29 & $\begin{array}{l}\text { C 74.85; } \\
\text { H 7.24; } \\
\text { N 7.32\% }\end{array}$ & $\mathrm{C}_{31} \mathrm{H}_{35} \mathrm{~N}_{3} \mathrm{O}_{3}$ & $\begin{array}{l}\text { C 74.82; } \\
\text { H 7.09; } \\
\text { N } 8.44 \%\end{array}$ \\
\hline
\end{tabular}




\begin{tabular}{|c|c|c|c|}
\hline 30 & $\begin{array}{l}\text { C 60.25; } \\
\text { H 7.34; } \\
\text { N 9.54\% }\end{array}$ & $\mathrm{C}_{30} \mathrm{H}_{36} \mathrm{~N}_{4} \mathrm{O} \bullet 2 \mathrm{HCl} \bullet 3 \mathrm{H}_{2} \mathrm{O}$ & $\begin{array}{l}\text { C 60.50; } \\
\text { Н 7.45; } \\
9.41 \%\end{array}$ \\
\hline 31 & $\begin{array}{l}\text { C 61.11; } \\
\text { H 8.17; } \\
\text { N 7.38\% }\end{array}$ & $\mathrm{C}_{31} \mathrm{H}_{35} \mathrm{~N}_{3} \mathrm{O}_{3} \bullet \mathrm{C}_{7} \mathrm{H}_{17} \mathrm{NO}_{5} \bullet 3.5 \mathrm{H}_{2} \mathrm{O}$ & $\begin{array}{l}\text { C } 60.38 ; \\
\text { H } 7.88 ; \\
\text { N } 7.41 \%\end{array}$ \\
\hline 32 & $\begin{array}{l}\text { C 65.20; } \\
\text { H 6.31; } \\
\text { N 6.78\% }\end{array}$ & $\mathrm{C}_{32} \mathrm{H}_{37} \mathrm{~N}_{3} \mathrm{O}_{3} \bullet \mathrm{C}_{2} \mathrm{HF}_{3} \mathrm{O}_{2}$ & $\begin{array}{l}\text { С 65.27; } \\
\text { H 6.12; } \\
\text { N } 6.72 \%\end{array}$ \\
\hline 33 & $\begin{array}{l}\text { C 61.60; } \\
\text { H 7.53; } \\
\text { N 7.81\% }\end{array}$ & $\mathrm{C}_{31} \mathrm{H}_{35} \mathrm{~N}_{3} \mathrm{O}_{3} \bullet \mathrm{C}_{7} \mathrm{H}_{17} \mathrm{NO}_{5} \bullet 2.5 \mathrm{H}_{2} \mathrm{O}$ & $\begin{array}{l}\text { C 61.85; } \\
\text { H 7.79; } \\
\text { N 7.59\% }\end{array}$ \\
\hline 34 & $\begin{array}{l}\text { C 58.94; } \\
\text { H 7.31; } \\
\text { N 7.60\% }\end{array}$ & $\mathrm{C}_{30} \mathrm{H}_{32} \mathrm{FN}_{3} \mathrm{O}_{3} \bullet \mathrm{C}_{7} \mathrm{H}_{17} \mathrm{NO}_{5} \bullet 3 \mathrm{H}_{2} \mathrm{O}$ & $\begin{array}{l}\text { C } 59.17 ; \\
\text { H } 7.38 ; \\
\text { N } 7.46 \%\end{array}$ \\
\hline 35 & $\begin{array}{l}\text { C 66.18; } \\
\text { H 7.69; } \\
\text { N 9.64\% }\end{array}$ & $\mathrm{C}_{32} \mathrm{H}_{38} \mathrm{~N}_{4} \mathrm{O}_{3} \bullet 3 \mathrm{H}_{2} \mathrm{O}$ & $\begin{array}{l}\text { C } 66.18 ; \\
\text { H } 7.64 ; \\
\text { N } 9.65 \%\end{array}$ \\
\hline 36 & $\begin{array}{l}\text { C 59.80; } \\
\text { H 7.65; } \\
\text { N 7.55\% }\end{array}$ & $\mathrm{C}_{31} \mathrm{H}_{35} \mathrm{~N}_{3} \mathrm{O}_{4} \bullet \mathrm{C}_{7} \mathrm{H}_{17} \mathrm{NO}_{5} \bullet 3 \mathrm{H}_{2} \mathrm{O}$ & $\begin{array}{l}\text { C 59.83; } \\
\text { H 7.66; } \\
\text { N } 7.34 \%\end{array}$ \\
\hline 37 & $\begin{array}{l}\text { C 61.40; } \\
\text { H 7.88; } \\
\text { N 7.65\% }\end{array}$ & $\mathrm{C}_{31} \mathrm{H}_{35} \mathrm{~N}_{3} \mathrm{O}_{3} \bullet \mathrm{C}_{7} \mathrm{H}_{17} \mathrm{NO}_{5} \bullet 2.7 \mathrm{H}_{2} \mathrm{O}$ & $\begin{array}{l}\text { C 61.51; } \\
\text { H 7.81; } \\
\text { N 7.55\% }\end{array}$ \\
\hline 38 & $\begin{array}{l}\text { C 61.03; } \\
\text { H 7.71; } \\
\text { N 7.40\% }\end{array}$ & $\mathrm{C}_{31} \mathrm{H}_{35} \mathrm{~N}_{3} \mathrm{O}_{3} \bullet \mathrm{C}_{7} \mathrm{H}_{17} \mathrm{NO}_{5} \bullet 3 \mathrm{H}_{2} \mathrm{O}$ & $\begin{array}{l}\text { C } 61.14 ; \\
\text { H 7.83; } \\
\text { N 7.51\% }\end{array}$ \\
\hline
\end{tabular}




\begin{tabular}{|c|c|c|c|}
\hline 3 & $\begin{array}{l}\text { C 62.32; } \\
\text { H 8.06; } \\
\text { N 7.31\% }\end{array}$ & $\mathrm{C}_{32} \mathrm{H}_{37} \mathrm{~N}_{3} \mathrm{O}_{3} \bullet \mathrm{C}_{7} \mathrm{H}_{17} \mathrm{NO}_{5} \bullet 2.5 \mathrm{H}_{2} \mathrm{O}$ & $\begin{array}{l}\text { C 62.28; } \\
\text { H 7.91; } \\
\text { N 7.45\% }\end{array}$ \\
\hline 40 & $\begin{array}{l}\text { C 62.94; } \\
\text { H 7.90; } \\
\text { N 7.51\% }\end{array}$ & $\mathrm{C}_{33} \mathrm{H}_{39} \mathrm{~N}_{3} \mathrm{O}_{3} \bullet \mathrm{C}_{7} \mathrm{H}_{17} \mathrm{NO}_{5} \bullet 2.3 \mathrm{H}_{2} \mathrm{O}$ & $\begin{array}{l}\text { C 63.10; } \\
\text { H 8.01; } \\
\text { N 7.36\% }\end{array}$ \\
\hline 41 & $\begin{array}{l}\text { C 53.10; } \\
\text { H 8.04; } \\
\text { N 8.39\% }\end{array}$ & $\mathrm{C}_{24} \mathrm{H}_{29} \mathrm{~N}_{3} \mathrm{O}_{3} \bullet \mathrm{C}_{7} \mathrm{H}_{17} \mathrm{NO}_{5} \bullet 5 \mathrm{H}_{2} \mathrm{O}$ & $\begin{array}{l}\text { C 53.74; } \\
\text { H 8.15; } \\
\text { N 8.09\% }\end{array}$ \\
\hline 42 & $\begin{array}{l}\text { C } 64.89 ; \\
\text { H } 7.41 ; \\
\text { N } 8.79 \%\end{array}$ & $\mathrm{C}_{26} \mathrm{H}_{33} \mathrm{~N}_{3} \mathrm{O}_{3} \bullet \mathrm{HCl} \bullet 0.5 \mathrm{H}_{2} \mathrm{O}$ & $\begin{array}{l}\text { C 64.96; } \\
\text { H 7.33; } \\
\text { N 8.74\% }\end{array}$ \\
\hline 43 & $\begin{array}{l}\text { C 57.52; } \\
\text { H 8.33; } \\
\text { N } 8.04 \%\end{array}$ & $\mathrm{C}_{26} \mathrm{H}_{33} \mathrm{~N}_{3} \mathrm{O}_{3} \bullet \mathrm{C}_{7} \mathrm{H}_{17} \mathrm{NO}_{5} \bullet 3.2 \mathrm{H}_{2} \mathrm{O}$ & $\begin{array}{l}\text { C 57.51; } \\
\text { H 8.26; } \\
\text { N 8.13\% }\end{array}$ \\
\hline 44 & $\begin{array}{l}\text { C 59.69; } \\
\text { H 8.46; } \\
\text { N 8.17\% }\end{array}$ & $\mathrm{C}_{27} \mathrm{H}_{35} \mathrm{~N}_{3} \mathrm{O}_{3} \bullet \mathrm{C}_{7} \mathrm{H}_{17} \mathrm{NO}_{5} \bullet 2.3 \mathrm{H}_{2} \mathrm{O}$ & $\begin{array}{l}\text { C 59.57; } \\
\text { H 8.31; } \\
\text { N 8.17\% }\end{array}$ \\
\hline 45 & $\begin{array}{l}\text { C 60.32; } \\
\text { H 8.01; } \\
\text { N 8.25\% }\end{array}$ & $\mathrm{C}_{26} \mathrm{H}_{31} \mathrm{~N}_{3} \mathrm{O}_{3} \bullet \mathrm{C}_{7} \mathrm{H}_{17} \mathrm{NO}_{5} \bullet 1.7 \mathrm{H}_{2} \mathrm{O}$ & $\begin{array}{l}\text { C 60.13; } \\
\text { H 7.86; } \\
\text { N 8.50\% }\end{array}$ \\
\hline 46 & $\begin{array}{l}\text { C 60.53; } \\
\text { H 8.39; } \\
\text { N 7.96\% }\end{array}$ & $\mathrm{C}_{28} \mathrm{H}_{35} \mathrm{~N}_{3} \mathrm{O}_{3} \bullet \mathrm{C}_{7} \mathrm{H}_{17} \mathrm{NO}_{5} \bullet 2.2 \mathrm{H}_{2} \mathrm{O}$ & $\begin{array}{l}\text { C 60.41; } \\
\text { H 8.16; } \\
\text { N 8.08\% }\end{array}$ \\
\hline 47 & $\begin{array}{l}\text { C 59.72; } \\
\text { H 8.33; } \\
\text { N 7.49\% }\end{array}$ & $\mathrm{C}_{29} \mathrm{H}_{37} \mathrm{~N}_{3} \mathrm{O}_{3} \bullet \mathrm{C}_{7} \mathrm{H}_{17} \mathrm{NO}_{5} \bullet 3.1 \mathrm{H}_{2} \mathrm{O}$ & $\begin{array}{l}\text { C 59.50; } \\
\text { H 8.35; } \\
\text { N 7.71\% }\end{array}$ \\
\hline
\end{tabular}




\begin{tabular}{|c|c|c|c|}
\hline 48 & $\begin{array}{l}\text { C 61.04; } \\
\text { H 8.49; } \\
\text { N 7.89\% }\end{array}$ & $\mathrm{C}_{30} \mathrm{H}_{39} \mathrm{~N}_{3} \mathrm{O}_{3} \bullet \mathrm{C}_{7} \mathrm{H}_{17} \mathrm{NO}_{5} \bullet 2.2 \mathrm{H}_{2} \mathrm{O}$ & $\begin{array}{l}\text { C } 61.23 \\
\text { H } 8.41 ; \\
\text { N } 7.72 \%\end{array}$ \\
\hline 49 & $\begin{array}{l}\text { C 60.54; } \\
\text { H 8.53; } \\
\text { N 7.48\% }\end{array}$ & $\mathrm{C}_{31} \mathrm{H}_{39} \mathrm{~N}_{3} \mathrm{O}_{3} \bullet \mathrm{C}_{7} \mathrm{H}_{17} \mathrm{NO}_{5} \bullet 3.0 \mathrm{H}_{2} \mathrm{O}$ & $\begin{array}{l}\text { C 60.78; } \\
\text { H 8.32; } \\
\text { N 7.46\% }\end{array}$ \\
\hline 50 & $\begin{array}{l}\text { C 63.15; } \\
\text { H 9.15; } \\
\text { N } 6.81 \%\end{array}$ & $\mathrm{C}_{35} \mathrm{H}_{49} \mathrm{~N}_{3} \mathrm{O}_{3} \bullet \mathrm{C}_{7} \mathrm{H}_{17} \mathrm{NO}_{5} \bullet 2.5 \mathrm{H}_{2} \mathrm{O}$ & $\begin{array}{l}\text { C 63.05; } \\
\text { H 8.96; } \\
\text { N 7.00\% }\end{array}$ \\
\hline 51 & $\begin{array}{l}\text { C 61.69; } \\
\text { H 8.56; } \\
\text { N 7.65\% }\end{array}$ & $\mathrm{C}_{30} \mathrm{H}_{39} \mathrm{~N}_{3} \mathrm{O}_{3} \bullet \mathrm{C}_{7} \mathrm{H}_{17} \mathrm{NO}_{5} \bullet 2 \mathrm{H}_{2} \mathrm{O}$ & $\begin{array}{l}\text { C 61.63; } \\
\text { H 8.39; } \\
\text { N 7.77\% }\end{array}$ \\
\hline 52 & $\begin{array}{l}\text { C 63.64; } \\
\text { H 8.72; } \\
\text { N 7.87\% }\end{array}$ & $\mathrm{C}_{31} \mathrm{H}_{41} \mathrm{~N}_{3} \mathrm{O}_{3} \bullet \mathrm{C}_{7} \mathrm{H}_{17} \mathrm{NO}_{5} \bullet 1.1 \mathrm{H}_{2} \mathrm{O}$ & $\begin{array}{l}\text { C 63.57; } \\
\text { H 8.44; } \\
\text { N 7.80\% }\end{array}$ \\
\hline 53 & $\begin{array}{l}\text { C 58.06; } \\
\text { H 8.12; } \\
\text { N 7.62\% }\end{array}$ & $\mathrm{C}_{28} \mathrm{H}_{35} \mathrm{~N}_{3} \mathrm{O}_{4} \bullet \mathrm{C}_{7} \mathrm{H}_{17} \mathrm{NO}_{5} \bullet 3 \mathrm{H}_{2} \mathrm{O}$ & $\begin{array}{l}\text { C 57.84; } \\
\text { H } 8.04 ; \\
\text { N } 7.71 \%\end{array}$ \\
\hline 54 & $\begin{array}{l}\text { C 60.50; } \\
\text { H 7.94; } \\
\text { N 7.49\% }\end{array}$ & $\mathrm{C}_{30} \mathrm{H}_{37} \mathrm{~N}_{3} \mathrm{O}_{4} \bullet \mathrm{C}_{7} \mathrm{H}_{17} \mathrm{NO}_{5} \bullet 2 \mathrm{H}_{2} \mathrm{O}$ & $\begin{array}{l}\text { C } 60.47 \\
\text { H 7.96; } \\
\text { N 7.62\% }\end{array}$ \\
\hline 55 & $\begin{array}{l}\text { C 62.29; } \\
\text { H 7.61; } \\
\text { N 7.45\% }\end{array}$ & $\mathrm{C}_{31} \mathrm{H}_{35} \mathrm{~N}_{3} \mathrm{O}_{4} \bullet \mathrm{C}_{7} \mathrm{H}_{17} \mathrm{NO}_{5} \bullet 1.2 \mathrm{H}_{2} \mathrm{O}$ & $\begin{array}{l}\text { C 62.56; } \\
\text { H 7.50; } \\
\text { N 7.68\% }\end{array}$ \\
\hline
\end{tabular}


${ }^{1}$ H NMR data of compounds used in biological tests

\begin{tabular}{|c|c|}
\hline Compound & Formula \\
\hline 14 & $\begin{array}{l}\delta 13.0(1 \mathrm{H}, \mathrm{br} \mathrm{s}), 11.22(1 \mathrm{H}, \mathrm{s}), 9.51(1 \mathrm{H}, \mathrm{s}), 8.32(1 \mathrm{H}, \mathrm{s}), 8.02 \\
(1 \mathrm{H}, \mathrm{d}), 7.60(1 \mathrm{H}, \mathrm{d}), 7.40(2 \mathrm{H}, \mathrm{m}), 7.25(3 \mathrm{H}, \mathrm{m}), 6.88(1 \mathrm{H}, \mathrm{s}), \\
2.95(2 \mathrm{H}, \mathrm{m}), 2.45(3 \mathrm{H}, \mathrm{s}), 1.94(3 \mathrm{H}, \mathrm{s}), 1.64(6 \mathrm{H}, \mathrm{m}), 1.53(6 \mathrm{H}, \\
\mathrm{s}), 1.36(2 \mathrm{H}, \mathrm{m}) .\end{array}$ \\
\hline 21 & $\begin{array}{l}\delta 12.72(1 \mathrm{H}, \mathrm{br} \mathrm{s}), 9.83(1 \mathrm{H}, \mathrm{s}), 8.50(1 \mathrm{H}, \mathrm{s}), 8.04(3 \mathrm{H}, \mathrm{m}), 7.63 \\
(1 \mathrm{H}, \mathrm{d}), 7.51-7.36(4 \mathrm{H}, \mathrm{m}), 2.99(2 \mathrm{H}, \mathrm{m}), 1.96(3 \mathrm{H}, \mathrm{br} \mathrm{s}), 1.66 \\
(6 \mathrm{H}, \mathrm{m}), 1.55(6 \mathrm{H}, \mathrm{s}), 1.43(2 \mathrm{H}, \mathrm{m}) .\end{array}$ \\
\hline 23 & $\begin{array}{l}\delta 12.52(1 \mathrm{H}, \mathrm{br} \mathrm{s}), 9.76(1 \mathrm{H}, \mathrm{s}), 8.49(1 \mathrm{H}, \mathrm{s}), 7.94(1 \mathrm{H}, \mathrm{m}), 7.60 \\
(2 \mathrm{H}, \mathrm{m}), 7.41(1 \mathrm{H}, \mathrm{t}), 7.32(3 \mathrm{H}, \mathrm{m}), 3.03(2 \mathrm{H}, \mathrm{m}), 2.54(3 \mathrm{H}, \mathrm{s}), \\
1.57(3 \mathrm{H}, \mathrm{m}), 0.93(6 \mathrm{H}, \mathrm{m}) .\end{array}$ \\
\hline 24 & $\begin{array}{l}\delta 12.53(1 \mathrm{H}, \mathrm{br} \mathrm{s}), 9.74(1 \mathrm{H}, \mathrm{s}), 8.47(1 \mathrm{H}, \mathrm{s}), 7.94(1 \mathrm{H}, \mathrm{m}), 7.60 \\
(2 \mathrm{H}, \mathrm{m}), 7.41(1 \mathrm{H}, \mathrm{t}), 7.32(3 \mathrm{H}, \mathrm{m}), 3.01(2 \mathrm{H}, \mathrm{m}), 2.55(3 \mathrm{H}, \mathrm{s}), \\
1.56(2 \mathrm{H}, \mathrm{m}), 0.95(9 \mathrm{H}, \mathrm{s}) .\end{array}$ \\
\hline 25 & $\begin{array}{l}\delta 12.50(1 \mathrm{H}, \mathrm{br} \mathrm{s}), 9.76(1 \mathrm{H}, \mathrm{s}), 8.49(1 \mathrm{H}, \mathrm{s}), 7.94(1 \mathrm{H}, \mathrm{m}), 7.60 \\
(2 \mathrm{H}, \mathrm{m}), 7.41(1 \mathrm{H}, \mathrm{t}), 7.33(3 \mathrm{H}, \mathrm{m}), 3.03(2 \mathrm{H}, \mathrm{m}), 2.54(3 \mathrm{H}, \mathrm{s}), \\
1.78-1.53(6 \mathrm{H}, \mathrm{m}), 1.18(5 \mathrm{H}, \mathrm{m}), 0.93(2 \mathrm{H}, \mathrm{m}) .\end{array}$ \\
\hline 26 & $\begin{array}{l}\delta 12.60(1 \mathrm{H}, \mathrm{br} \mathrm{s}), 9.79(1 \mathrm{H}, \mathrm{s}), 8.51(1 \mathrm{H}, \mathrm{s}), 7.95(1 \mathrm{H}, \mathrm{m}), 7.60 \\
(2 \mathrm{H}, \mathrm{m}), 7.42(1 \mathrm{H}, \mathrm{m}), 7.32(8 \mathrm{H}, \mathrm{m}), 3.31(2 \mathrm{H}, \mathrm{m}), 2.99(2 \mathrm{H}, \\
\mathrm{m}), 2.51(3 \mathrm{H}, \mathrm{s}) .\end{array}$ \\
\hline 27 & $\begin{array}{l}\delta 12.36(1 \mathrm{H}, \mathrm{br} \mathrm{s}), 9.81(1 \mathrm{H}, \mathrm{s}), 8.51(1 \mathrm{H}, \mathrm{s}), 7.91(1 \mathrm{H}, \mathrm{d}), 7.60 \\
(2 \mathrm{H}, \mathrm{m}), 7.40(1 \mathrm{H}, \mathrm{t}), 7.32(3 \mathrm{H}, \mathrm{m}), 2.84(2 \mathrm{H}, \mathrm{s}), 2.53(3 \mathrm{H}, \mathrm{s}), \\
1.91(3 \mathrm{H}, \mathrm{br} \mathrm{s}), 1.65-1.56(12 \mathrm{H}, \mathrm{m}) .\end{array}$ \\
\hline 28 & $\begin{array}{l}\delta 9.13(2 \mathrm{H}, \mathrm{br} \mathrm{s}), 7.57(2 \mathrm{H}, \mathrm{m}), 7.46(1 \mathrm{H}, \mathrm{m}), 7.35-7.19(4 \mathrm{H}, \mathrm{m}), \\
6.92(1 \mathrm{H}, \mathrm{d}), 3.16(2 \mathrm{H}, \mathrm{m}), 2.60(3 \mathrm{H}, \mathrm{s}), 2.36(3 \mathrm{H}, \mathrm{s}), 1.99(3 \mathrm{H}, \\
\mathrm{s}), 1.75-1.56(12 \mathrm{H}, \mathrm{m}), 1.51-1.45(2 \mathrm{H}, \mathrm{m}) .\end{array}$ \\
\hline 29 & $\begin{array}{l}\delta 9.46(1 \mathrm{H}, \mathrm{br} \mathrm{s}), 9.23(1 \mathrm{H}, \mathrm{s}), 8.14(1 \mathrm{H}, \mathrm{s}), 8.01(1 \mathrm{H}, \mathrm{m}), 7.70 \\
(1 \mathrm{H}, \mathrm{m}), 7.50(1 \mathrm{H}, \mathrm{d}), 7.35(1 \mathrm{H}, \mathrm{t}), 7.28-7.21(3 \mathrm{H}, \mathrm{m}), 3.85(3 \mathrm{H}, \\
\mathrm{s}), 3.07(2 \mathrm{H}, \mathrm{m}), 2.54(3 \mathrm{H}, \mathrm{s}), 1.91(3 \mathrm{H}, \mathrm{s}), 1.68-1.41(14 \mathrm{H}, \mathrm{m}) .\end{array}$ \\
\hline 31 & $\begin{array}{l}\delta 12.70(1 \mathrm{H}, \mathrm{br} \mathrm{s}), 9.55(1 \mathrm{H}, \text { br s}), 7.70(1 \mathrm{H}, \mathrm{s}), 7.61(2 \mathrm{H}, \mathrm{m}), \\
7.30(4 \mathrm{H}, \mathrm{m}), 6.94(1 \mathrm{H}, \mathrm{d}), 3.54(2 \mathrm{H}, \mathrm{s}), 2.98(2 \mathrm{H}, \mathrm{m}), 1.95(3 \mathrm{H}, \\
\text { br s), } 1.65(6 \mathrm{H}, \mathrm{m}), 1.54(6 \mathrm{H}, \mathrm{s}), 1.43(2 \mathrm{H}, \mathrm{m}) .\end{array}$ \\
\hline
\end{tabular}




\begin{tabular}{|c|c|}
\hline 33 & $\begin{array}{l}\delta 12.80(1 \mathrm{H}, \text { br s), } 12.49(1 \mathrm{H}, \text { br s }), 9.62(1 \mathrm{H}, \text { br s }), 8.31(1 \mathrm{H}, \\
\mathrm{m}), 7.79(1 \mathrm{H}, \mathrm{m}), 7.58(1 \mathrm{H}, \mathrm{m}), 7.33-7.20(4 \mathrm{H}, \mathrm{m}), 2.98(2 \mathrm{H}, \\
\mathrm{m}), 2.54(3 \mathrm{H}, \mathrm{s}), 2.46(3 \mathrm{H}, \mathrm{s}), 1.95(3 \mathrm{H}, \mathrm{s}), 1.67-1.40(14 \mathrm{H}, \mathrm{m}) .\end{array}$ \\
\hline 34 & $\begin{array}{l}\delta 12.50(1 \mathrm{H}, \text { br s }), 10.27(1 \mathrm{H}, \text { br s }), 8.38(1 \mathrm{H}, \mathrm{m}), 7.98(1 \mathrm{H}, \mathrm{m}), \\
7.63(1 \mathrm{H}, \mathrm{d}), 7.45-7.25(4 \mathrm{H}, \mathrm{m}), 3.00(2 \mathrm{H}, \mathrm{m}), 2.52(3 \mathrm{H}, \mathrm{s}), 1.95 \\
(3 \mathrm{H}, \mathrm{s}), 1.65(6 \mathrm{H}, \mathrm{m}), 1.55(6 \mathrm{H}, \mathrm{br} \mathrm{s}), 1.44(2 \mathrm{H}, \mathrm{m}) .\end{array}$ \\
\hline 35 & $\begin{array}{l}\delta 12.51(1 \mathrm{H}, \text { br s }), 9.80(1 \mathrm{H}, \mathrm{s}), 8.52(1 \mathrm{H}, \mathrm{d}), 7.99(1 \mathrm{H}, \mathrm{dd}), 7.72 \\
(1 \mathrm{H}, \mathrm{d}), 7.59(1 \mathrm{H}, \mathrm{d}), 7.30(3 \mathrm{H}, \mathrm{m}), 2.96(2 \mathrm{H}, \mathrm{m}), 2.81(6 \mathrm{H}, \mathrm{s}), \\
2.54(3 \mathrm{H}, \mathrm{s}), 1.95(3 \mathrm{H}, \text { br s }), 1.75-1.46(14 \mathrm{H}, \mathrm{m}) .\end{array}$ \\
\hline 36 & $\begin{array}{l}\delta 12.50(1 \mathrm{H}, \text { br s), } 9.55(1 \mathrm{H}, \text { br s), } 8.11(1 \mathrm{H}, \mathrm{d}), 7.81(1 \mathrm{H}, \mathrm{dd}) \text {, } \\
7.59(1 \mathrm{H}, \mathrm{d}), 7.30(3 \mathrm{H}, \mathrm{m}), 7.07(1 \mathrm{H}, \mathrm{d}), 3.78(3 \mathrm{H}, \mathrm{s}), 2.98(2 \mathrm{H} \text {, } \\
\text { m) } 2.54(3 \mathrm{H}, \mathrm{s}), 1.95(3 \mathrm{H}, \mathrm{s}), 1.71-1.40(14 \mathrm{H}, \mathrm{m}) .\end{array}$ \\
\hline 37 & $\begin{array}{l}\delta 13.00(1 \mathrm{H}, \text { br s }), 12.43(1 \mathrm{H}, \mathrm{s}), 9.71(1 \mathrm{H}, \mathrm{s}), 8.47(1 \mathrm{H}, \mathrm{s}), 7.94 \\
(1 \mathrm{H}, \mathrm{d}), 7.62(1 \mathrm{H}, \mathrm{d}), 7.44(2 \mathrm{H}, \mathrm{m}), 7.12(2 \mathrm{H}, \mathrm{m}), 2.97(2 \mathrm{H}, \mathrm{m}), \\
2.52(3 \mathrm{H}, \mathrm{s}), 2.31(3 \mathrm{H}, \mathrm{s}), 1.95(3 \mathrm{H}, \mathrm{br} \mathrm{s}), 1.66(6 \mathrm{H}, \mathrm{m}), 1.54 \\
(6 \mathrm{H}, \mathrm{s}), 1.42(2 \mathrm{H}, \mathrm{m}) .\end{array}$ \\
\hline 38 & $\begin{array}{l}\delta 12.43(1 \mathrm{H}, \mathrm{s}), 9.82(1 \mathrm{H}, \mathrm{s}), 8.53(1 \mathrm{H}, \mathrm{s}), 7.92(1 \mathrm{H}, \mathrm{d}), 7.60 \\
(1 \mathrm{H}, \mathrm{d}), 7.38(1 \mathrm{H}, \mathrm{m}), 7.26(1 \mathrm{H}, \mathrm{m}), 7.14(2 \mathrm{H}, \mathrm{d}), 3.00(2 \mathrm{H}, \mathrm{m}), \\
2.11(6 \mathrm{H}, \mathrm{s}), 1.95(3 \mathrm{H}, \mathrm{s}), 1.65(6 \mathrm{H}, \mathrm{m}), 1.51(6 \mathrm{H}, \mathrm{s}), 1.43(2 \mathrm{H}, \\
\text { m). }\end{array}$ \\
\hline 39 & $\begin{array}{l}\delta 12.36(1 \mathrm{H}, \text { br s }), 9.80(1 \mathrm{H}, \mathrm{s}), 8.52(1 \mathrm{H}, \mathrm{s}), 7.92(1 \mathrm{H}, \mathrm{d}), 7.60 \\
(1 \mathrm{H}, \mathrm{d}), 7.38(1 \mathrm{H}, \mathrm{t}), 6.95(2 \mathrm{H}, \mathrm{s}), 2.99(2 \mathrm{H}, \mathrm{m}), 2.28(3 \mathrm{H}, \mathrm{s}), \\
2.07(6 \mathrm{H}, \mathrm{s}), 1.93(3 \mathrm{H}, \mathrm{s}), 1.63(6 \mathrm{H}, \mathrm{m}), 1.51(6 \mathrm{H}, \mathrm{s}), 1.43(2 \mathrm{H}, \\
\text { m). }\end{array}$ \\
\hline 40 & $\begin{array}{l}\delta 12.75(1 \mathrm{H}, \text { br s }), 12.33(1 \mathrm{H}, \text { br s }), 9.68(1 \mathrm{H}, \mathrm{s}), 8.35(1 \mathrm{H}, \mathrm{d}), \\
7.77(1 \mathrm{H}, \mathrm{dd}), 7.18(1 \mathrm{H}, \mathrm{d}), 6.95(2 \mathrm{H}, \mathrm{s}), 2.98(2 \mathrm{H}, \mathrm{m}), 2.45(3 \mathrm{H}, \\
\mathrm{s}), 2.28(3 \mathrm{H}, \mathrm{s}), 2.06(6 \mathrm{H}, \mathrm{s}), 1.93(3 \mathrm{H}, \mathrm{s}), 1.63(6 \mathrm{H}, \mathrm{m}), 1.51 \\
(6 \mathrm{H}, \mathrm{s}), 1.42(2 \mathrm{H}, \mathrm{m}) .\end{array}$ \\
\hline 41 & $\begin{array}{l}\delta 10.60(1 \mathrm{H}, \mathrm{s}), 8.40(1 \mathrm{H}, \mathrm{s}), 7.96(1 \mathrm{H}, \mathrm{m}), 7.69(1 \mathrm{H}, \mathrm{m}), 7.48 \\
(1 \mathrm{H}, \mathrm{t}), 2.92(2 \mathrm{H}, \mathrm{m}), 2.55(3 \mathrm{H}, \mathrm{s}), 1.94(3 \mathrm{H}, \mathrm{br} \mathrm{s}), 1.64(6 \mathrm{H}, \mathrm{m}), \\
1.50(6 \mathrm{H}, \mathrm{m}), 1.38(2 \mathrm{H}, \mathrm{m}) .\end{array}$ \\
\hline 42 & $\begin{array}{l}\delta 8.35(1 \mathrm{H}, \mathrm{m}), 7.81(1 \mathrm{H}, \mathrm{m}), 7.48(1 \mathrm{H}, \mathrm{t}), 2.99(2 \mathrm{H}, \mathrm{m}), 2.83 \\
(2 \mathrm{H}, \mathrm{t}), 1.96(3 \mathrm{H}, \mathrm{s}), 1.79(8 \mathrm{H}, \mathrm{m}), 1.59(6 \mathrm{H}, \mathrm{m}), 1.44(2 \mathrm{H}, \mathrm{m}), \\
1.02(3 \mathrm{H}, \mathrm{t}) .\end{array}$ \\
\hline 43 & $\begin{array}{l}\delta 13.20(1 \mathrm{H}, \text { br s }), 12.25(1 \mathrm{H}, \text { br s}), 9.77(1 \mathrm{H}, \text { br s }), 8.45(1 \mathrm{H}, \mathrm{s}), \\
7.91(1 \mathrm{H}, \mathrm{m}), 7.61(1 \mathrm{H}, \mathrm{d}), 7.41(1 \mathrm{H}, \mathrm{t}), 3.10(1 \mathrm{H}, \mathrm{m}), 2.90(2 \mathrm{H}, \\
\mathrm{m}), 1.94(3 \mathrm{H}, \text { br s}), 1.63(6 \mathrm{H}, \mathrm{m}), 1.51(6 \mathrm{H}, \mathrm{s}), 1.36(2 \mathrm{H}, \mathrm{m}),\end{array}$ \\
\hline
\end{tabular}




\begin{tabular}{|c|c|}
\hline & $1.29(3 \mathrm{H}, \mathrm{s}), 1.27(3 \mathrm{H}, \mathrm{s})$ \\
\hline 44 & $\begin{array}{l}\text { S } 8.36(1 \mathrm{H}, \mathrm{t}), 7.93(1 \mathrm{H}, \mathrm{dd}), 7.76(1 \mathrm{H}, \mathrm{dd}), 7.45(1 \mathrm{H}, \mathrm{t}), 2.98 \\
(2 \mathrm{H}, \mathrm{m}), 1.96(3 \mathrm{H}, \mathrm{s}), 1.72(6 \mathrm{H}, \mathrm{m}), 1.60(6 \mathrm{H}, \mathrm{m}), 1.41(11 \mathrm{H}, \\
\mathrm{m}) .\end{array}$ \\
\hline 45 & $\begin{array}{l}\delta 9.51(1 \mathrm{H}, \mathrm{s}), 8.39(1 \mathrm{H}, \mathrm{s}), 7.85(1 \mathrm{H}, \mathrm{d}), 7.60(1 \mathrm{H}, \mathrm{d}), 7.34(1 \mathrm{H}, \\
\mathrm{t}), 2.86(2 \mathrm{H}, \mathrm{m}), 1.94(4 \mathrm{H}, \mathrm{m}), 1.64(6 \mathrm{H}, \mathrm{m}), 1.50(6 \mathrm{H}, \mathrm{m}), 1.33 \\
(2 \mathrm{H}, \mathrm{m}), 0.89(4 \mathrm{H}, \mathrm{m}) .\end{array}$ \\
\hline 46 & $\begin{array}{l}\delta 12.00(1 \mathrm{H}, \mathrm{br} \mathrm{s}), 9.59(1 \mathrm{H}, \mathrm{s}), 8.45(1 \mathrm{H}, \mathrm{s}), 7.89(1 \mathrm{H}, \mathrm{dd}), 7.59 \\
(1 \mathrm{H}, \mathrm{d}), 7.38(1 \mathrm{H}, \mathrm{t}), 3.02(1 \mathrm{H}, \mathrm{m}), 2.87(2 \mathrm{H}, \mathrm{m}), 1.94(5 \mathrm{H}, \mathrm{m}), \\
1.77-1.58(12 \mathrm{H}, \mathrm{m}), 1.50(6 \mathrm{H}, \mathrm{s}), 1.34(2 \mathrm{H}, \mathrm{m}) .\end{array}$ \\
\hline 47 & $\begin{array}{l}\delta 13.00(1 \mathrm{H}, \mathrm{br} \mathrm{s}), 11.94(1 \mathrm{H}, \mathrm{br} \mathrm{s}), 9.57(1 \mathrm{H}, \mathrm{s}), 8.46(1 \mathrm{H}, \mathrm{s}), \\
7.88(1 \mathrm{H}, \mathrm{d}), 7.58(1 \mathrm{H}, \mathrm{d}), 7.39(1 \mathrm{H}, \mathrm{t}), 2.88(2 \mathrm{H}, \mathrm{m}), 2.60(1 \mathrm{H}, \\
\mathrm{m}), 1.93-1.26(27 \mathrm{H}, \mathrm{m}) .\end{array}$ \\
\hline 48 & $\begin{array}{l}\text { S } 8.36(1 \mathrm{H}, \mathrm{t}), 7.91(1 \mathrm{H}, \mathrm{dt}), 7.79(1 \mathrm{H}, \mathrm{dt}), 7.44(1 \mathrm{H}, \mathrm{t}), 2.97 \\
(2 \mathrm{H}, \mathrm{m}), 2.90(1 \mathrm{H}, \mathrm{m}), 1.97(6 \mathrm{H}, \mathrm{m}), 1.87-1.60(23 \mathrm{H}, \mathrm{m}), 1.41 \\
(2 \mathrm{H}, \mathrm{m}) .\end{array}$ \\
\hline 49 & $\begin{array}{l}\delta 11.86(1 \mathrm{H}, \mathrm{br} \mathrm{s}), 9.49(1 \mathrm{H}, \mathrm{br} \mathrm{s}), 8.43(1 \mathrm{H}, \mathrm{s}), 7.89(1 \mathrm{H}, \mathrm{d}), \\
7.59(1 \mathrm{H}, \mathrm{d}), 7.40(1 \mathrm{H}, \mathrm{t}), 2.86(2 \mathrm{H}, \mathrm{m}), 1.93(3 \mathrm{H}, \mathrm{s}), 1.80(6 \mathrm{H}, \\
\mathrm{m}), 1.64(13 \mathrm{H}, \mathrm{m}), 1.50(6 \mathrm{H}, \mathrm{s}), 1.32(2 \mathrm{H}, \mathrm{m}\end{array}$ \\
\hline 50 & $\begin{array}{l}\delta 12.50(1 \mathrm{H}, \mathrm{br} \mathrm{s}), 12.00(1 \mathrm{H}, \mathrm{s}), 9.58(1 \mathrm{H}, \mathrm{s}), 8.45(1 \mathrm{H}, \mathrm{s}), 7.89 \\
(1 \mathrm{H}, \mathrm{d}), 7.59(1 \mathrm{H}, \mathrm{d}), 7.38(1 \mathrm{H}, \mathrm{t}), 2.88(3 \mathrm{H}, \mathrm{m}), 1.93(3 \mathrm{H}, \mathrm{s}), \\
1.70-1.30(36 \mathrm{H}, \mathrm{m}) .\end{array}$ \\
\hline 51 & $\begin{array}{l}\text { 813.00 }(1 \mathrm{H}, \text { br s), } 11.85(1 \mathrm{H}, \mathrm{br} \mathrm{s}), 9.54(1 \mathrm{H}, \mathrm{br} \mathrm{s}), 8.40(1 \mathrm{H}, \mathrm{s}), \\
7.92(1 \mathrm{H}, \mathrm{d}), 7.61(1 \mathrm{H}, \mathrm{d}), 7.42(1 \mathrm{H}, \mathrm{t}), 2.90(2 \mathrm{H}, \mathrm{m}), 2.12(2 \mathrm{H}, \\
\mathrm{m}), 1.93(3 \mathrm{H}, \mathrm{br} \mathrm{s}), 1.63(6 \mathrm{H}, \mathrm{m}), 1.51-1.33(16 \mathrm{H}, \mathrm{m}), 1.22(3 \mathrm{H}, \\
\text { s). }\end{array}$ \\
\hline 52 & $\begin{array}{l}\delta 11.80(1 \mathrm{H}, \mathrm{br} \mathrm{s}), 9.36(1 \mathrm{H}, \mathrm{s}), 8.21(1 \mathrm{H}, \mathrm{d}), 7.75(1 \mathrm{H}, \mathrm{dd}), 7.19 \\
(1 \mathrm{H}, \mathrm{d}), 2.87(2 \mathrm{H}, \mathrm{m}), 2.45(3 \mathrm{H}, \mathrm{s}), 2.11(2 \mathrm{H}, \mathrm{m}), 1.93(3 \mathrm{H}, \mathrm{br} \\
\mathrm{s}), 1.62(6 \mathrm{H}, \mathrm{m}), 1.50-1.32(16 \mathrm{H}, \mathrm{m}), 1.20(3 \mathrm{H}, \mathrm{s}) .\end{array}$ \\
\hline 54 & $\begin{array}{l}\delta 12.80(1 \mathrm{H}, \mathrm{br} \mathrm{s}), 12.06(1 \mathrm{H}, \mathrm{br} \mathrm{s}), 9.59(1 \mathrm{H}, \mathrm{s}), 8.42(1 \mathrm{H}, \mathrm{s}), \\
7.92(1 \mathrm{H}, \mathrm{d}), 7.60(1 \mathrm{H}, \mathrm{d}), 7.41(1 \mathrm{H}, \mathrm{t}), 4.77(2 \mathrm{H}, \mathrm{s}), 2.10(3 \mathrm{H}, \\
\text { br s), } 1.84(6 \mathrm{H}, \mathrm{m}), 1.76(6 \mathrm{H}, \mathrm{m}), 1.64-1.53(13 \mathrm{H}, \mathrm{m}) .\end{array}$ \\
\hline 55 & $\begin{array}{l}\delta 12.50(1 \mathrm{H}, \mathrm{br} \mathrm{s}), 9.92(1 \mathrm{H}, \mathrm{s}), 8.53(1 \mathrm{H}, \mathrm{s}), 7.94(1 \mathrm{H}, \mathrm{m}), 7.62 \\
(1 \mathrm{H}, \mathrm{d}), 7.40(1 \mathrm{H}, \mathrm{t}), 6.95(2 \mathrm{H}, \mathrm{s}), 4.91(2 \mathrm{H}, \mathrm{s}), 2.28(3 \mathrm{H}, \mathrm{s}), \\
2.10(3 \mathrm{H}, \mathrm{s}), 2.07(6 \mathrm{H}, \mathrm{s}), 1.77(6 \mathrm{H}, \mathrm{s}), 1.57(6 \mathrm{H}, \mathrm{m})\end{array}$ \\
\hline
\end{tabular}


